OPEN ACCESS

Edited by: Puneeth lyengar,

University of Texas Southwestern Medical Center, United States

Reviewed by: Alessandro de Sire, University of Magna Graecia, Italy

Ester Orlandi,

National Center of Oncological Hadrontherapy, Italy

${ }^{*}$ Correspondence:

Daniel Clayburgh clayburg@ohsu.edu Aaron J. Grossberg grossber@ohsu.edu

Specialty section: This article was submitted to Cancer Metabolism, a section of the journa

Frontiers in Oncology

Received: 09 November 2021 Accepted: 24 January 2022 Published: 14 February 2022

Citation:

Olson B, Edwards J, Degnin C, Santucci N, Buncke M, Hu J, Chen Y, Fuller $C D$, Geltzeiler $M$, Grossberg AJ and Clayburgh D (2022) Establishment and Validation of Pre-Therapy Cervical Vertebrae Muscle Quantification as a

Prognostic Marker of Sarcopenia in Patients With Head and Neck Cancer.

Front. Oncol. 12:812159. doi: 10.3389/fonc.2022.812159

\section{Establishment and Validation of Pre- Therapy Cervical Vertebrae Muscle Quantification as a Prognostic Marker of Sarcopenia in Patients With Head and Neck Cancer}

\author{
Brennan Olson ${ }^{1,2}$, Jared Edwards ${ }^{3}$, Catherine Degnin ${ }^{4}$, Nicole Santucci ${ }^{1}$, \\ Michelle Buncke ${ }^{1}$, Jeffrey $\mathrm{Hu}^{1}$, Yiyi Chen ${ }^{5}$, Clifton D. Fuller ${ }^{6}$, Mathew Geltzeiler ${ }^{7}$, \\ Aaron J. Grossberg ${ }^{8,9,10^{*}}$ and Daniel Clayburgh ${ }^{7,11^{*}}$ \\ 1 School of Medicine, Oregon Health \& Science University, Portland, OR, United States, ${ }^{2}$ Medical Scientist Training Program, \\ Oregon Health \& Science University, Portland, OR, United States, ${ }^{3}$ Department of General Surgery, Naval Medical Center San \\ Diego, San Diego, CA, United States, ${ }^{4}$ Biostatistics Shared Resources, Knight Cancer Institute, Oregon Health \& Science \\ University, Portland, OR, United States, ${ }^{5}$ Seagen Inc., Bothell, WA, United States, ${ }^{6}$ Department of Radiation Oncology, The \\ University of Texas MD Anderson Cancer Center, Houston, TX, United States, 7 Department of Otolaryngology/Head and Neck \\ Surgery, Oregon Health \& Science University, Portland, OR, United States, ${ }^{8}$ Department of Radiation Medicine, Oregon Health \& \\ Science University, Portland, OR, United States, ${ }^{9}$ Brenden-Colson Center for Pancreatic Care, Oregon Health \& Science \\ University, Portland, OR, United States, ${ }^{10}$ Cancer Early Detection Advanced Research Center, Knight Cancer Institute, Oregon \\ Health \& Science University, Portland, OR, United States, ${ }^{11}$ Operative Care Division, Portland Veterans Affairs Health Care \\ System, Portland, OR, United States
}

Background: Sarcopenia is prognostic for survival in patients with head and neck cancer (HNC). However, identification of this high-risk feature remains challenging without computed tomography $(\mathrm{CT})$ imaging of the abdomen or thorax. Herein, we establish sarcopenia thresholds at the C3 level and determine if C3 sarcopenia is associated with survival in patients with HNC.

Methods: This retrospective cohort study was conducted in consecutive patients with a squamous cell carcinoma of the head and neck with cross-sectional abdominal or neck imaging within 60 days prior to treatment. Measurement of cross-sectional muscle area at L3 and C3 levels was performed from CT imaging. Primary study outcome was overall survival.

Results: Skeletal muscle area at C3 was strongly correlated with the L3 level in both men ( $\mathrm{n}=$ 188; $r=0.77 ; p<0.001)$ and women $(n=65 ; r=0.80 ; p<0.001)$, and C3 sarcopenia thresholds of $14.0 \mathrm{~cm}^{2} / \mathrm{m}^{2}$ (men) and $11.1 \mathrm{~cm}^{2} / \mathrm{m}^{2}$ (women) were best predictive of L3 sarcopenia thresholds. Applying these C3 thresholds to a cohort of patients with neck imaging alone revealed that C3 sarcopenia was independently associated with reduced overall survival in men $(H R=2.63 ; 95 \% \mathrm{Cl}, 1.79,3.85)$ but not women $(H R=1.18,95 \% \mathrm{Cl}, 0.76,1.85)$.

Conclusions: This study identifies sarcopenia thresholds at the C3 level that best predict L3 sarcopenia in men and women. In HNC, C3-defined sarcopenia is associated with poor survival outcomes in men, but not women, suggesting sarcopenia may differentially affect men and women with HNC.

Keywords: head and neck cancer, squamous cell carcinoma, surgery, sarcopenia, cachexia, muscle wasting, body composition 


\section{INTRODUCTION}

Patients with cancer frequently experience weight loss, including progressive lean and fat mass catabolism consistent with the paraneoplastic wasting syndrome of cachexia $(1,2)$. Excessive skeletal muscle wasting, or sarcopenia, is significantly associated with morbidity and mortality for patients with solid tumors (35). Sarcopenia can exist as an isolated finding as is often the case in the elderly or secondary to a disease process that activates catabolic programs-including cancer $(1,6)$. While sarcopenia is richly described as a negative prognostic marker for patients with primary tumors in the abdomen, only recently has its importance been identified in patients with head and neck cancer (HNC) (79). Indeed, sarcopenia is a significant predictor of survival and post-operative complications for patients with HNC and shows promise as a risk-stratification tool for patients with this disease (7-10). Furthermore, sarcopenia is significantly associated with the development of cancer-associated fatigue in patients with head and neck cancer, and improvement of muscle mass during disease progression through resistance training may be beneficial in mitigating fatigue and improve overall quality of life (11-13). Therefore, regular detection of sarcopenia in patients with head and neck cancer may allow for early intervention and riskstratification, which could improve patients' quality of life and ultimate survival. Despite the clear utility in identifying sarcopenia in patients with HNC, clinical implementation of sarcopenia measures remains challenging due to the lack of established approaches that integrate with standard clinical workflow (14).

In terms of clinical detection, dual-energy $\mathrm{x}$-ray absorptiometry was initially used in detecting sarcopenia, but the most widely utilized method today includes identification of cross-sectional skeletal muscle area from computed tomography (CT) scans (15). However, several alternative methods exist, including bioelectrical impedance analysis, ultrasound of the tibialis anterior, magnetic resonance imaging, and serum biomarker analysis (14, 16-18). Despite the advent of newer detection methods, CT-defined muscle wasting remains highly utilized for several reasons, including its relative ease of analysis compared to alternative methods, and its potential for automation and integration into the electronic health record $(19,20)$. Since imaging studies (CT or $\mathrm{PET} / \mathrm{CT}$ ) are routinely performed during the clinical workup of HNC, establishing cross-sectional skeletal muscle area thresholds utilizing these scans represent a low-cost, reliable, and reproducible method for determining sarcopenia in this patient population. Current skeletal muscle index (SMI, defined as crosssectional skeletal muscle area normalized to patients' body height squared) values for defining sarcopenia are specific to abdominal or lower thoracic musculature $(5,21)$. As abdominal and lower thoracic imaging is not always performed as part of the HNC workup, only a subset of patients that have imaging studies capturing these regions are included in most previous reports, ultimately introducing selection bias. Therefore, defining and validating sarcopenia thresholds from routinely acquired head and neck images would greatly enhance clinical implementation while allowing better cross-study comparisons. Previous reports demonstrated a significant correlation between cervical and abdominal vertebrae cross sectional muscle area in patients with $\mathrm{HNC}$, suggesting that routine head and neck imaging may be used to identify sarcopenia (22-26). In the present study, we sought to expand upon these findings in a cohort of HNC patients treated with primary surgical resection by defining sex-specific sarcopenia thresholds, evaluating their prognostic value, and validating these associations in an independent patient cohort.

\section{METHODS}

\section{Population Cohort and End Points}

We performed a retrospective review by screening medical records of patients who underwent primary surgical resection of head and neck squamous cell carcinoma between January 1, 2005 and December 21, 2017 at Oregon Health and Science University in Portland, Oregon. Electronic health records were reviewed for data collection and included: patient demographics, body mass and height, comorbidities, tumor staging and subsite information, $\mathrm{HPV} / \mathrm{p} 16$ status, smoking status, treatment information, evidence of recurrence, date and cause of death, and date of last follow up. Patients were classified as underweight (BMI <18.5), normal weight (BMI 18.5-24.9), overweight (BMI 25-29.9) or obese (BMI >30). Smoking status was binned in 3 groups, including never smokers, $<10$ pack years, $\geq 10$ pack years. Feeding tube classifications included patients never receiving a G-tube, those receiving a G-tube temporary, and those who had feeding tubes in situ at the time of last follow up. Tumor sites included: oropharynx, oral cavity, larynx, and other. Pathologic $\mathrm{T}$ staging was binned as either T0-2 or T3-4, while pathologic $\mathrm{N}$ staging was binned as either N0-1, N2-3, or NX. Charlson Comorbidity Index (CCI) score was calculated as previously described (27) and patients were subsequently stratified as either low risk $(\mathrm{CCI}<5)$ or high risk $(\mathrm{CCI} \geq 5)$. After patient data were abstracted and coupled to their imaging information, all patient data were de-identified for subsequent analyses. This study was approved by the Institutional Review Board at Oregon Health and Science University. Requirement for informed consent was waived due to the retrospective nature of this study.

We utilized two separate cohorts of patients with HNC for analyses. The first cohort, defined as the training cohort, is utilized to establish C3 sarcopenia thresholds and contains patients that have CT imaging that captures both the L3 and C3 vertebral levels. The second cohort, defined as the validation cohort, consists of HNC patients with only neck imaging (skull base to lung apices) and is utilized for validation of these established C3 thresholds on mortality outcomes. Study inclusion for both training and validation cohorts was restricted to patients treated with primary surgical resection of head and neck squamous cell carcinoma at Oregon Health and Science University between January 2005 and December 2017 with PET/CT or CT scans within 60 days prior to surgical resection. Therefore, all patients' scans utilized in this study were capturing muscle area prior to surgery and/or adjuvant therapy. For the training cohort, patients were required to have a scan that captured both the C3 and L3 vertebral levels, while the validation cohort only required capture of the C3 level. 


\section{Computed Tomography Body Composition Analysis}

Body composition analysis of skeletal muscle was performed as previously described in patients with $\operatorname{HNC}(9,22)$. Briefly, the cross-sectional area of skeletal muscle at the center of the third lumbar (L3) and third cervical (C3) vertebrae was determined by segmentation of axial CT images (Figure 1). Segmentation analysis was performed using Slice-o-Matic Software (version 5.0; Tomovision; https://www.tomovision.com) to define muscle tissue cross-sectional area. Muscles delineated in the segmentation analysis included the rectus abdominus, abdominal wall, psoas, and the paraspinal muscle groups at the L3 level. Muscles measured at $\mathrm{C} 3$ included the paraspinal muscle group and the sternocleidomastoid (SCM). Muscle tissue was defined as -29 to 150 Hounsfield units as described previously (28). The resulting cross-sectional muscle area was then normalized to the square of the patient's height in meters and used to calculate skeletal muscle index (SMI). At the L3 level, sarcopenia is defined as an SMI of less than $52.4 \mathrm{~cm}^{2} / \mathrm{m}^{2}$ for men and $38.5 \mathrm{~cm}^{2} / \mathrm{m}^{2}$ for women (5). These thresholds are consistent with previous reports in head and neck cancer patients.

\section{Statistical Analysis}

To compare patient and clinical-disease characteristics at baseline, the Chi-square or Fisher's Exact tests were used for categorical variables; the Wilcoxon rank sum test was used for continuous variables. Data was presented as median (IQR) or frequency $(\%)$. Training cohort $(n=253)$ comparisons were stratified by L3-defined sarcopenia; validation cohort $(n=536)$ comparisons were stratified by C3-defined sarcopenia.

Correlation was assessed using Pearson's rho, which assumes normal distribution and a linear relationship between the measurements. Rho greater than 0.8 is considered 'very strong' correlation; rho between 0.6-0.79 suggests 'strong' correlation. To test for linearity and homoscedasticity we plotted residuals versus fitted values, showing the loess (smoothing) curve in red. Receiver Operating Characteristic (ROC) curves were generated to show the general predictive ability of C3 to predict L3-defined sarcopenia; DeLong's test of correlated ROC curves was used to discriminate between C3 measurement types (29). We used Youden's Index to determine the optimal C3 cut-off value for predicting sarcopenia.

Overall survival is the time from initial diagnosis until death by any cause, with participants censored at their last assessment date. Assumptions of proportionality in the survival models were verified graphically and using residual-based models. Univariate and multivariable Cox proportional hazard ratio models were used to assess the risk of death based on demographic and baseline clinical-disease characteristics. We used purposeful selection combined with Bayesian information criterion (BIC) to build the multivariable models, entering all variables from the univariate models with $\mathrm{p}$-value $<0.2$. Kaplan-Meier curves with log-rank test were used to display overall survival stratified by C3-defined sarcopenia. All analyses were conducted using R, version 3.5.3.

\section{RESULTS}

\section{Determination of C3-Defined Sarcopenia Thresholds}

The training cohort included patients with both abdominal and neck imaging in order to perform intrapatient correlative analyses of L3 and C3 SMI values to identify appropriate C3 sarcopenia thresholds. Median age of this cohort is 61 (IQR 54, 68 ) years with $188 / 253(74 \%)$ patients identifying as male. In this cohort, $6.0 \%$ of patients were underweight (BMI <18.5), 32\% were normal weight (BMI 18.5-24.9), 38\% were overweight (BMI 25-29.9), and 24\% were obese (BMI >30). Eighty-nine patients were never smokers (35\%), 51 (20\%) patients smoked $<10$ pack years, and 113 (45\%) patients had a $\geq 10$ pack year history. Postoperatively, 130 (51\%) patients received temporary feeding tubes while 47 (19\%) patients still had feeding tubes in situ at the time of last follow up. The majority of this cohort had oropharynx disease (147 patients [58\%]), while primary tumors in the oral cavity (55 [22\%]) and larynx (19 [8\%]) were less frequently observed (27). The majority of patients were classified as lowrisk by the Charlson Comorbidity Index (CCI <5; 213 [84\%]).
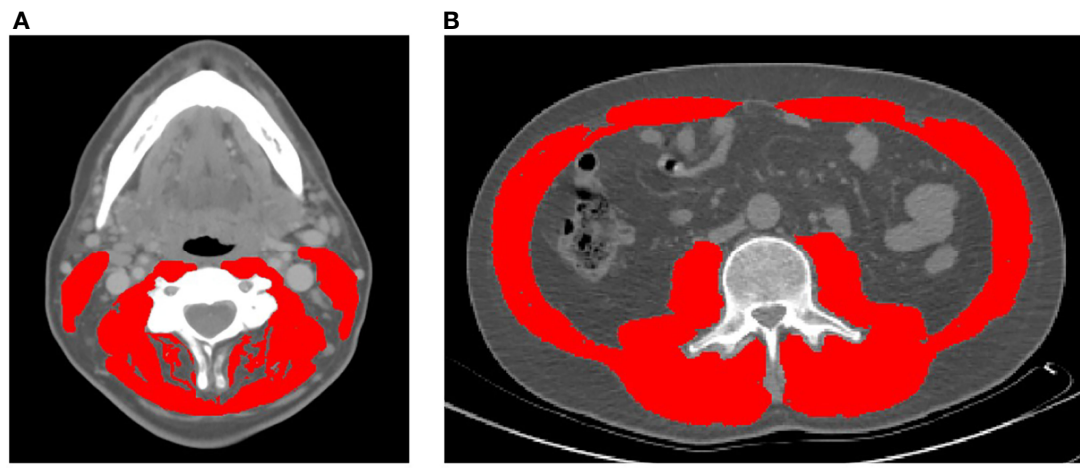

FIGURE 1 | Representative axial CT images of the third cervical (A) and third lumbar (B) vertebral levels used to quantify skeletal muscle index (highlighted in red). 
One hundred three (41\%) patients were treated by primary surgical resection alone, while 150 (59\%) patients received adjuvant therapy (radiation and/or chemotherapy). Full characteristics and details of this cohort stratified by L3-defined sarcopenia are shown in eTable $\mathbf{1}$ in the Supplement.

Intrapatient L3 and C3 levels were strongly correlated in both men $(\mathrm{n}=188, \mathrm{r}=0.77 ; \mathrm{p}<0.001)$ and women $(\mathrm{n}=65, \mathrm{r}=0.80$; $\mathrm{p}<0.001$; Figure 2; Supplementary Figure 1). As it is possible for the borders of the SCM to be obscured by lymph node metastases, we performed comparative analyses amongst L3 and C3 SMI values, both inclusive and exclusive of the SCM, to examine whether inclusion of the SCM improves or worsens predictive capacity of L3-based sarcopenia. Including the SCM in C3 SMI measurements improved predictive capacity of L3defined sarcopenia in women (AUC $=89.4 \%$ vs. $86.3 \%$; $\mathrm{p}=$ 0.03 ), but not men (AUC $=85.9 \%$ vs. $85.0 \% ; \mathrm{p}=0.30$; Supplementary Figure 2). Therefore, we included the SCM in all subsequent analyses. The C3 SMI thresholds with the best model performance based on Youden's Index were $14.0 \mathrm{~cm}^{2} / \mathrm{m}^{2}$ for men and $11.1 \mathrm{~cm}^{2} / \mathrm{m}^{2}$ for women (Figure 2). Finally, we confirmed the utility of C3-defined sarcopenia in this training cohort through survival analysis which demonstrated an association between C3-defined sarcopenia in men ( $\mathrm{HR}=1.9$; 95\% CI, 1.2-5.5), but not women ( $\mathrm{HR}=0.84$; 95\% CI, 0.30-2.15), with HNC (Supplementary Figure 3).

\section{Survival Analyses in C3-Defined Sarcopenic Patients}

We next applied these C3 SMI thresholds to an independent cohort of HNC patients with imaging studies (PET/CT or CT) that captured the head and neck, but not abdomen or lower thorax (Table 1). This validation cohort included patients with imaging of the head and neck, but not abdomen or lower thorax. Median age of patients in this cohort was 64 (IQR 56, 72) with 333 patients identifying as male (62\%) and 203 identifying as female (38\%; Table 1). Forty-one (7.7\%) patients were underweight, 211 (40\%) were normal weight, 164 (31\%) were overweight, and $118(22 \%)$ were classified as obese (BMI >30). This cohort included 162 (30\%) never smokers, 68 (13\%) patients with $<10$ pack years, and $306(57 \%)$ patients with $\geq 10$ pack years. Postoperatively, 174 (32\%) patients received temporary feeding tubes and 105 (20\%) patients still had feeding tubes at last follow up. In contrast to the training cohort, the preponderance of this cohort had oral cavity disease (306 patients [57\%]), while primary tumors in the oropharynx (118 [22\%]) and larynx (79 [15\%]) were less frequently observed. Using CCI, 409 [76\%] patients were classified as low risk and 127 were classified as [24\%] high risk. In the validation cohort, $324(60 \%)$ patients were treated by primary surgical resection alone, with 212 (40\%) receiving adjuvant therapy (radiation and/or chemotherapy). Baseline patient characteristics stratified by C3-defined sarcopenia are shown in Table 1.

In this cohort, $53 \%(175 / 333)$ of men were classified as sarcopenic compared to just 38\% (77/203) of women (Table 1 and eTable 2). Univariate Cox proportional hazards analysis revealed advanced age at time of surgery, C3-defined sarcopenia, underweight BMI status, $>10$ pack-year smoking status, permanent feeding tube placement, negative HPV status, elevated CCI, pT3-4, pN2-3, group stage 4, angiolymphatic invasion (ALI), perineural invasion (PNI), and extranodal extension (ENE) as significant predictors of survival in men. Univariate cox modelling for variables associated with survival in women included permanent feeding tube placement, elevated CCI, T category (pT3-4), group stage 3 and 4, ALI, and PNI (eTable3). Kaplan-Meier survival curves showed a significant difference in survival based on C3-defined sarcopenia for men but not women (Figure 3). On multivariable analyses, C3defined sarcopenia $(\mathrm{HR}=2.67 ; 95 \% \mathrm{CI}, 1.72-4.15)$, ALI $(\mathrm{HR}=$ 2.00; 95\% CI, 1.32-3.02), permanent feeding tube placement $(\mathrm{HR}=2.33 ; 95 \% \mathrm{CI}, 1.41-3.88)$, and age at time of surgery $(\mathrm{HR}=1.04 ; 95 \% \mathrm{CI}, 1.02-1.05)$ all remained significantly associated with overall survival for men (Table 2). In women, PNI ( $\mathrm{HR}=2.45 ; 95 \% \mathrm{CI}, 1.48-4.06)$, ALI ( $\mathrm{HR}=2.13 ; 95 \% \mathrm{CI}$, 1.22-3.72), and pathologic $\mathrm{T}$ category ( $\mathrm{HR}=1.95$; $95 \% \mathrm{CI}, 1.20$ 3.16) were associated with reduced overall survival on multivariable analysis (Table 2). In the training cohort, advanced age was associated with sarcopenia in women, while advanced age was associated with sarcopenia in men in the validation cohort (eTable4).
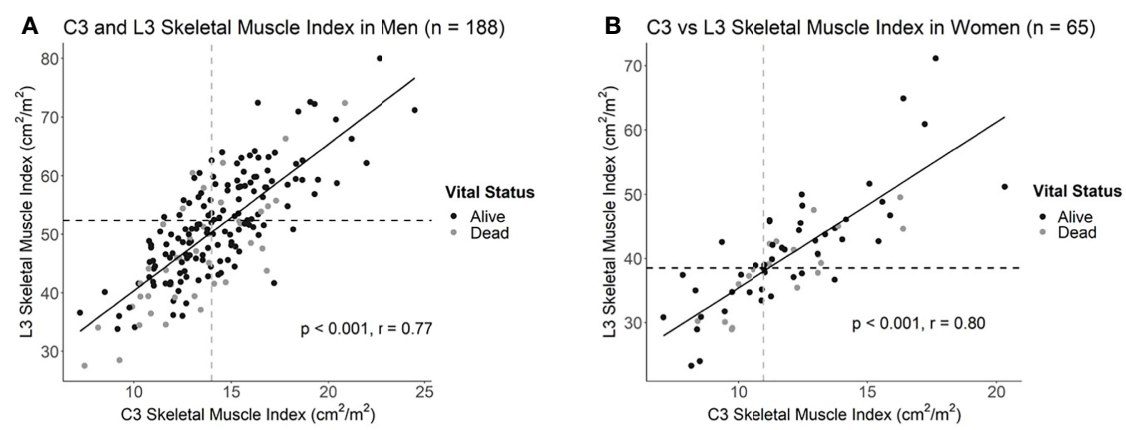

FIGURE 2 | Correlation plots of L3 and C3 skeletal muscle indices in (A) men and (B) women with head and neck cancer. Dotted lines delineate the intersection of previously established L3 sarcopenia thresholds with the estimated C3 sarcopenia thresholds. 
TABLE 1 | Clinical-demographic tables for validation set, stratified by C3-defined sarcopenia (Men: C3<14.0; Women: C3<11.1; n = 536).

\begin{tabular}{|c|c|c|c|c|}
\hline Risk Factor & Overall, $\mathrm{N}=536^{1}$ & Sarcopenic, $N=252^{1}$ & Not sarcopenic, $N=284^{1}$ & p-value ${ }^{2}$ \\
\hline Age at time of surgery & $64(56,72)$ & $65(58,71)$ & $62(55,72)$ & 0.200 \\
\hline RT Fractions & $33(30,35)$ & $33(30,35)$ & $32(30,35)$ & 0.028 \\
\hline (Missing) & 344 & 166 & 178 & \\
\hline RT dose & $6,300(6,000,6,600)$ & $6,300(6,000,6,650)$ & $6,000(6,000,6,600)$ & 0.140 \\
\hline (Missing) & 338 & 163 & 175 & \\
\hline Days from Diagnosis to surgery & $31(21,42)$ & $34(21,44)$ & $29(20,41)$ & 0.090 \\
\hline (Missing) & 10 & 6 & 4 & \\
\hline Vital Status & & & & $<0.001$ \\
\hline Alive & $332(62 \%)$ & $134(53 \%)$ & $198(70 \%)$ & \\
\hline Dead & $204(38 \%)$ & $118(47 \%)$ & $86(30 \%)$ & \\
\hline Sex & & & & 0.001 \\
\hline Male & $333(62 \%)$ & $175(69 \%)$ & $158(56 \%)$ & \\
\hline Female & $203(38 \%)$ & $77(31 \%)$ & $126(44 \%)$ & \\
\hline BMI & & & & $<0.001$ \\
\hline Underweight & $41(8 \%)$ & $33(13 \%)$ & $8(3 \%)$ & \\
\hline Normal weight & $211(40 \%)$ & $126(50 \%)$ & $85(30 \%)$ & \\
\hline Overweight & $164(31 \%)$ & $71(28 \%)$ & $93(33 \%)$ & \\
\hline Obese & $118(22 \%)$ & $21(8 \%)$ & $97(34 \%)$ & \\
\hline (Missing) & 2 & 1 & 1 & \\
\hline Smoking Status & & & & 0.003 \\
\hline Never smoke & $162(30 \%)$ & 59 (23\%) & $103(36 \%)$ & \\
\hline <10 pack years & $68(13 \%)$ & $31(12 \%)$ & $37(13 \%)$ & \\
\hline >= 10 pack years & $306(57 \%)$ & $162(64 \%)$ & $144(51 \%)$ & \\
\hline Feeding Tube & & & & 0.053 \\
\hline No G-tube & $257(48 \%)$ & $107(42 \%)$ & $150(53 \%)$ & \\
\hline Temporary G-tube & $174(32 \%)$ & $92(37 \%)$ & 82 (29\%) & \\
\hline Permanent G-tube & $105(20 \%)$ & $53(21 \%)$ & $52(18 \%)$ & \\
\hline HPV & & & & 0.055 \\
\hline$H P V-$ & 99 (57\%) & $48(66 \%)$ & $51(50 \%)$ & \\
\hline$H P V_{+}$ & $76(43 \%)$ & $25(34 \%)$ & $51(50 \%)$ & \\
\hline (Missing) & 361 & 179 & 182 & \\
\hline Charlson Comorbidity Score & & & & 0.900 \\
\hline$<5$ & 409 (76\%) & $191(76 \%)$ & $218(77 \%)$ & \\
\hline$>=5$ & $127(24 \%)$ & $61(24 \%)$ & $66(23 \%)$ & \\
\hline Tumor site & & & & 0.130 \\
\hline Oral cavity & $306(57 \%)$ & $146(58 \%)$ & $160(56 \%)$ & \\
\hline Oropharynx & $118(22 \%)$ & $48(19 \%)$ & $70(25 \%)$ & \\
\hline Larynx & 79 (15\%) & 37 (15\%) & $42(15 \%)$ & \\
\hline Other & $33(6 \%)$ & $21(8 \%)$ & $12(4 \%)$ & \\
\hline Pathologic T category (pT) & & & & 0.002 \\
\hline TO-2 & $354(68 \%)$ & $149(61 \%)$ & $205(74 \%)$ & \\
\hline T3-4 & $170(32 \%)$ & 97 (39\%) & $73(26 \%)$ & \\
\hline (Missing) & 12 & 6 & 6 & \\
\hline Pathologic $\mathrm{N}$ category (pN) & & & & 0.500 \\
\hline NO-1 & $348(66 \%)$ & $161(65 \%)$ & $187(67 \%)$ & \\
\hline N2-3 & $151(29 \%)$ & 71 (29\%) & 80 (29\%) & \\
\hline$x$ & $26(5 \%)$ & $15(6 \%)$ & $11(4 \%)$ & \\
\hline (Missing) & 11 & 5 & 6 & \\
\hline Group stage & & & & 0.024 \\
\hline Stage 1 & $145(28 \%)$ & $60(24 \%)$ & 85 (31\%) & \\
\hline Stage 2 & $109(21 \%)$ & $42(17 \%)$ & 67 (24\%) & \\
\hline Stage 3 & $83(16 \%)$ & $43(17 \%)$ & $40(14 \%)$ & \\
\hline Stage 4 & $187(36 \%)$ & $101(41 \%)$ & $86(31 \%)$ & \\
\hline (Missing) & 12 & 6 & 6 & \\
\hline Margins status & & & & 0.500 \\
\hline Margins- & $424(84 \%)$ & $201(86 \%)$ & $223(83 \%)$ & \\
\hline Margins+ & $78(16 \%)$ & $33(14 \%)$ & 45 (17\%) & \\
\hline (Missing) & 34 & 18 & 16 & \\
\hline ALI & & & & 0.800 \\
\hline Absent & $324(66 \%)$ & $152(66 \%)$ & $172(67 \%)$ & \\
\hline Present & $139(28 \%)$ & $65(28 \%)$ & $74(29 \%)$ & \\
\hline Indeterminate/Suspicious & $26(6 \%)$ & $14(6 \%)$ & $12(5 \%)$ & \\
\hline (Missing) & 47 & 21 & 26 & \\
\hline
\end{tabular}


TABLE 1 | Continued

\begin{tabular}{|c|c|c|c|c|}
\hline PNI & & & & 0.400 \\
\hline Absent & $344(70 \%)$ & 158 (68\%) & $186(72 \%)$ & \\
\hline Present & 142 (29\%) & 72 (31\%) & 70 (27\%) & \\
\hline Suspicious & $4(1 \%)$ & $1(0.4 \%)$ & $3(1 \%)$ & \\
\hline (Missing) & 46 & 21 & 25 & \\
\hline ENE & & & & 0.300 \\
\hline Absent & 402 (83\%) & 185 (81\%) & 217 (85\%) & \\
\hline Present & 80 (17\%) & 43 (19\%) & 37 (15\%) & \\
\hline (Missing) & 54 & 24 & 30 & \\
\hline Recurrence & & & & 0.130 \\
\hline No recurrence & 402 (75\%) & $181(72 \%)$ & 221 (78\%) & \\
\hline Recurrence & $134(25 \%)$ & $71(28 \%)$ & 63 (22\%) & \\
\hline RT & & & & $>0.9$ \\
\hline No $R T$ & 325 (61\%) & 153 (61\%) & 172 (61\%) & \\
\hline$R T$ & 211 (39\%) & 99 (39\%) & 112 (39\%) & \\
\hline Chemotherapy & & & & 0.800 \\
\hline No Chemo & 437 (82\%) & 207 (82\%) & 230 (81\%) & \\
\hline Chemo & 99 (18\%) & 45 (18\%) & 54 (19\%) & \\
\hline Treatment Group & & & & $>0.9$ \\
\hline Surgery & 324 (60\%) & 152 (60\%) & 172 (61\%) & \\
\hline Surgery + Adjuvant & 212 (40\%) & 100 (40\%) & 112 (39\%) & \\
\hline
\end{tabular}

${ }^{1}$ Statistics presented: Median (IQR); $n$ (\%).

${ }^{2}$ Statistical tests performed: Wilcoxon rank-sum test; chi-square test of independence; Fisher's exact test.

Bold text indicates $p<.05$.
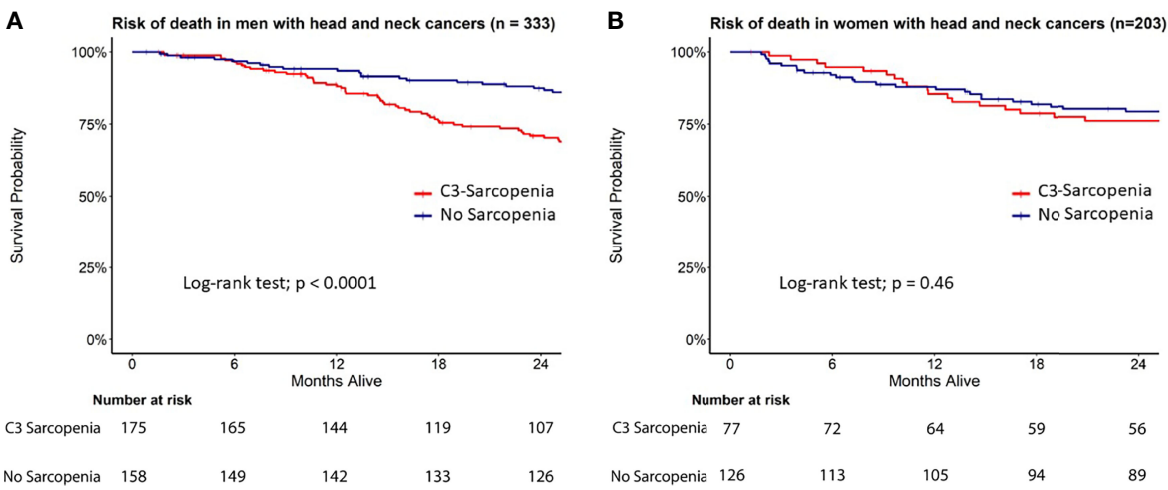

FIGURE 3 | Survival analysis in C3-defined sarcopenic male and female HNC patients. Kaplan Meier curves for (A) men and (B) women stratified by C3 sarcopenia.

\section{DISCUSSION}

Skeletal muscle depletion is a well-established prognostic marker in multiple clinical and disease settings, including cancer $(7,8)$, trauma (30), and drug dose scaling to minimize toxicities (31). Previous reports demonstrate the prognostic utility of sarcopenia is independent of patient body mass $(5,8,32)$, and that height and weight formulae are not sufficient to capture sarcopenia (33). However, identification of this high-risk disease feature is challenging in HNC because imaging studies that capture the abdomen or lower thorax are infrequent in this population. Although previous studies demonstrate promise in utilizing neck imaging as a marker of sarcopenia $(22,25,26,34)$, we sought to expand upon these foundational studies by putting forth normalized sex-specific sarcopenia thresholds and testing their prognostic utility in an independent cohort of patients with neck imaging alone. To our knowledge, our study is the first to establish normalized sex-specific C3 sarcopenia thresholds and validate their prognostic value in an independent cohort of HNC patients with imaging studies specific to the neck. We found that these C3 SMI thresholds were prognostic in men, but not women, with HNC.

Several foundational studies demonstrated a strong correlation of $\mathrm{C} 3$ and $\mathrm{L} 3$ skeletal muscle area in patients with HNC consistent with our findings herein (22, 23, 25, 35-37). One report utilized a single SMI cut-off for both men and women, an approach that may not accurately account for intrinsic differences in body habitus between male and female sexes (36). A recent study by Ufuk and colleagues found that among the $\mathrm{C} 2-\mathrm{C} 4$ vertebral levels, the paraspinal muscles at $\mathrm{C} 3$ were the most predictive of L3 in males, while summative C2-C4 SCM measurements were the most discriminative in females (23). 
TABLE 2 | Multivariable model for factors associated with risk of death with head and neck cancers in validation cohort, by sex ( $\mathrm{n}=536$ ).

\begin{tabular}{|c|c|c|c|c|c|c|}
\hline \multirow[t]{2}{*}{ Variables: } & \multicolumn{3}{|c|}{ Model selected for Men $(n=333)$} & \multicolumn{3}{|c|}{ Model selected for Women $(n=203)$} \\
\hline & $H^{1}$ & $95 \% \mathrm{Cl}^{1}$ & p-value & $\mathrm{HR}^{1}$ & $95 \% \mathrm{Cl}^{1}$ & p-value \\
\hline C3-Sarcopenia & & & $<0.001$ & & & \\
\hline Not sarcopenic & - & - & & & & \\
\hline Sarcopenic & 2.67 & $1.72,4.15$ & & & & \\
\hline ALI & & & 0.001 & & & 0.011 \\
\hline Absent & - & - & & - & - & \\
\hline Present & 2.00 & 1.32, 3.02 & & 2.13 & $1.22,3.72$ & \\
\hline Feeding Tube & & & 0.002 & & & \\
\hline No G-tube & - & - & & & & \\
\hline Temporary G-tube & 1.09 & $0.66,1.81$ & & & & \\
\hline Permanent G-tube & 2.33 & $1.41,3.88$ & & & & \\
\hline Age at time of surgery & 1.04 & $1.02,1.05$ & $<0.001$ & & & \\
\hline PNI & & & & & & $<0.001$ \\
\hline Absent & & & & - & - & \\
\hline Present & & & & 2.45 & $1.48,4.06$ & \\
\hline Pathologic T category (pT) & & & & & & 0.009 \\
\hline TO-2 & & & & - & - & \\
\hline T3-4 & & & & 1.95 & $1.20,3.16$ & \\
\hline
\end{tabular}

${ }^{1} \mathrm{HR}$, Hazard Ratio; Cl, Confidence Interval.

Bold text indicates $p<.05$.

However, a combinatorial approach in which both paraspinal and SCM muscle groups was not utilized in this report, and it stands to reason that increasing the measurable skeletal muscle area at the cervical level would only serve to increase the predictive capacity for sarcopenia at the lumbar level, as our data herein suggest (Supplementary Figure 2). Another recent report investigating the predictive capacity of C3 SMI on L3 SMI in a Korean HNC population demonstrated significant correlation in non-sarcopenic patients, but no such correlation in sarcopenic patients (defined as a calculated L3 muscle index of $<55 \mathrm{~cm}^{2} / \mathrm{m}^{2}$ in men and $<39 \mathrm{~cm}^{2} / \mathrm{m}^{2}$ in women) (38). In the present study, we observe significant correlation for both nonsarcopenic and sarcopenic HNC patients. Possible explanations for these differences in results could be due to the population of study or techniques in measuring skeletal muscle area (38). Specifically, we included both paraspinal and SCM muscles when measuring C3 SMI due to its improved predictive capacity of L3 (Supplementary Figure 2); we also utilized previously published L3 sarcopenia thresholds of $<52.4 \mathrm{~cm}^{2} / \mathrm{m}^{2}$ for men and $<38.5 \mathrm{~cm}^{2} / \mathrm{m}^{2}$ for women $(23,38)$. Similarly, in another recent report utilizing cross-sectional muscle area at the C3 level to predict L3 cross-sectional area, the authors classified sarcopenia as the lowest quartile of predicted L3 muscle area, while the work herein sought to base C3 sarcopenia thresholds on L3 sarcopenia thresholds that are validated across multiple cancers and conditions (25). Accordingly, it is possible that these C3 sarcopenia thresholds extend to other diseases and clinical settings in which the abdomen or lower thorax are not routinely captured by CT imaging, such as neurologic cancers. However, identifying the most suitable sarcopenia thresholds appears to be multifactorial and dependent on sex and disease-specific conditions (39), further highlighting the need for validating sarcopenia thresholds in a context-specific manner.

In the context of this recent literature, we believe our work adds substantially to this area of research for several reasons:
(1) through the largest cohort study to date, we further evidence that muscle area at the $\mathrm{C} 3$ vertebral level is closely predictive of the L3 level in both sarcopenic and non-sarcopenic HNC patients; (2) we establish that measuring both paraspinal and SCM musculature is more predictive than paraspinal SMI calculations alone, as many previous studies utilize only paraspinal muscles for sarcopenia measurements; (3) we establish normalized sex-specific C3 thresholds that are bestpredictive of widely published L3 sarcopenia thresholds, rather than utilizing ad hoc thresholds that are predictive of a single cohort; (4) we validate the prognostic utility of our proposed C3 sarcopenia thresholds in an independent cohort of patients with only neck CT imaging available; and (5) finally, we are the first to demonstrate a sexually-dimorphic survival outcome for HNC patients with sarcopenia, as sarcopenia was associated with reduced survival in men, but not women $(25,39)$. To our knowledge, this is the largest dataset of women with HNC stratified by sarcopenia, and the first to individually assess sexspecific survival outcomes during this disease.

Like many research areas, sarcopenia has largely been studied in males in both murine and clinical settings, while few studies evaluating sex as a biological variable exist (40,41). Amongst experimental rodent models of colon- and HNC-associated cachexia, male mice lost a larger proportion of lean mass compared to female mice with similar disease burden $(42,43)$. Several studies also report a significantly lower prevalence of sarcopenia in women with various cancers, including non-small cell lung and gastrointestinal cancers $(44,45)$. In the present study, we observe $55 \%$ of men are sarcopenic at the time of diagnosis compared to just $39 \%$ of women ( $p<0.001$ ). In addition to reports that describe a decrease in the prevalence of sarcopenia in women at the time of cancer diagnosis, Kilgour and colleagues showed a strong association between muscle mass and cancer-associated fatigue in men, but not in women (46). Burkart and colleagues recently showed an association between 
sarcopenia and overall survival in men with aggressive B-cell Non-Hodgkin Lymphoma, while women demonstrated no such association (47). Conversely, sarcopenia was associated with poorer survival outcomes in women with non-metastatic breast cancer (32). These studies and more suggest that sarcopenia may not only disproportionately appear between men and women during disease, but also differentially influence quality of life and mortality in a sex- and disease-specific manner (39).

It is plausible that the sarcopenia observed in this population is associated with cachexia, a disease-associated metabolic syndrome that significantly reduces patient's quality of life and ultimate survival (1). In the present study, it is possible that sarcopenia portends the development of cachexia more frequently in men than women, or that muscle loss is not associated with impaired resilience in women with $\mathrm{HNC}$ - future prospective investigations are needed to test these hypotheses. While this study demonstrates pre-therapy C3-defined sarcopenia is a useful prognostic marker in men, but not women, diagnosed with HNC, a recent report demonstrated that conversion to sarcopenia (detected by L3 muscle area) was associated with a reduction in overall survival in patients with HNC treated with definitive RT (7). Therefore, the sex-specific influence of post-therapy conversion to sarcopenia using the C3-sarcopenia thresholds established herein remains an area of active investigation. Collectively, our findings highlight the need for future studies to be deliberate in examining sex-specific effects and prevalence of sarcopenia.

\section{Limitations}

This study has several limitations that should be taken into consideration when interpreting these data. As with any retrospective review, this study is subjected to missing data and heterogeneous patient follow-up. This study was performed at a single tertiary care institution, resulting in a patient demographic that may not be reflective of other areas of the country. Finally, given the timeframe of this study that traverses the recognition of $\mathrm{HPV} / \mathrm{p} 16$ as a prognostic factor, these patients are staged by the American Joint Committee on Cancer (AJCC) seventh edition, as opposed AJCC eight edition staging criteria.

C3 thresholds were not developed against the 'gold standard' for sarcopenia, either volumetric full body cross sectional imaging or dual x-ray absorptiometry. A limitation of this study is that a surrogate marker of sarcopenia, normalized L3 skeletal muscle cross sectional area, is used to estimate the C3 threshold value and may imprecisely estimate sarcopenia. However, this measure is associated with survival endpoints in multiple disease states, supporting its use in the present study. We directly tested C3 measurements with risk of death using a partitioning approach (partDSA survival package in R) which generated a similar threshold value for men but provided no distinct C3 cut-off value for women.

\section{CONCLUSIONS}

Taken together, this study demonstrates that C3-sarcopenia thresholds are strongly associated with previously defined L3 sarcopenia thresholds, and C3-defined sarcopenia is independently associated with reduced survival in men, but not women with HNC. We propose that the C3-defined sarcopenia thresholds herein represent a useful prognostic tool for men with HNC. Future research concerning these observations is warranted, including determining whether these thresholds and sex-specific survival associations extend to other pathologies.

\section{DATA AVAILABILITY STATEMENT}

The raw data supporting the conclusions of this article will be made available by the authors, without undue reservation.

\section{ETHICS STATEMENT}

This study was approved by the Institutional Review Board at Oregon Health and Science University. Written informed consent for participation was not required for this study in accordance with the national legislation and the institutional requirements.

\section{AUTHOR CONTRIBUTIONS}

Conceptualization: BO, AG, and DC. Data curation: BO, JE, NS, $\mathrm{MB}$, and JH. Formal analysis: $\mathrm{CD}$ and YC. Funding acquisition: $\mathrm{BO}$ and $\mathrm{AG}$. Investigation: $\mathrm{BO}, \mathrm{CDF}, \mathrm{MG}, \mathrm{AG}$, and $\mathrm{DC}$. Methodology: BO, JE, CD, and YC. Resources: BO, CD, CDF, MG, AG, and DC. Software: BO and AG. Supervision: CDF, MG, AG, and DC. Writing - original draft: BO, AG, and DC. Writing review and editing: $\mathrm{BO}, \mathrm{JE}, \mathrm{CD}, \mathrm{NS}, \mathrm{MB}, \mathrm{JH}, \mathrm{YC}, \mathrm{CDF}, \mathrm{MG}, \mathrm{AG}$, and DC. All authors contributed to the article and approved the submitted version.

\section{ACKNOWLEDGMENTS}

This work was supported National Cancer Institute grants CA254033 (BO) and CA245188 (AG), AACR The Mark Foundation "Science of the Patient" Award 20-60-51-MARK (AG and Daniel L. Marks), as well as RSNA Research Medical Student Grant RMS2026 (BO). CF received/receives funding and salary support during the period of study execution unrelated to this work from: the National Institutes of Health (NIH) National Cancer Institute (NCI) Early Stage Development of Technologies in Biomedical Computing, Informatics, and Big Data Science Program (R01CA214825); Joint NSF/NIH Initiative on Quantitative Approaches to Biomedical Big Data program (R01CA225190); NIH National Institute of Biomedical Imaging and Bioengineering (NIBIB) Research Education Programs for Residents and Clinical Fellows Grant (R25EB025787); NIH National Institute of Dental and Craniofacial Research (NIDCR) Academic Industrial Partnership Grant (R01DE028290); NIDCR Establishing Outcome Measures for Clinical Studies of Oral and Craniofacial Diseases and Conditions award (R01DE025248); NCI Parent Research Project Grant (R01CA258827); NCI Early Phase 
Clinical Trials in Imaging and Image-Guided Interventions Program (1R01CA218148); an NIH/NCI Cancer Center Support Grant (CCSG) Pilot Research Program Award from the UT MD Anderson CCSG Radiation Oncology and Cancer Imaging Program (P30CA016672); a sub-award from the Small Business Innovation Research Grant Program (R43CA254559); a subaward from the The Human BioMolecular Atlas Program (HuBMAP) Integration, Visualization \& Engagement (HIVE) Initiative (OT2OD026675); the Patient-Centered Outcomes Research Institute (PCS-1609-36195); a National Science Foundation (NSF) Division of Civil, Mechanical, and Manufacturing Innovation (CMMI) grant (NSF 1933369); and the NSF/NCI Smart connected Health Program (R01CA257814). $\mathrm{CF}$ receives infrastructure support from MD Anderson Cancer

\section{REFERENCES}

1. Olson B, Marks DL, Grossberg AJ. Diverging Metabolic Programmes and Behaviours During States of Starvation, Protein Malnutrition, and Cachexia. J Cachexia Sarcopenia Muscle (2020) 11(6):1429-46. doi: 10.1002/jcsm.12630

2. Baracos VE, Martin L, Korc M, Guttridge DC, Fearon KCH. CancerAssociated Cachexia. Nat Rev Dis Primers (2018) 4:17105. doi: 10.1038/ nrdp.2017.105

3. Rinninella E, Cintoni M, Raoul P, Pozzo C, Strippoli A, Bria E, et al. Muscle Mass, Assessed at Diagnosis by L3-CT Scan as a Prognostic Marker of Clinical Outcomes in Patients With Gastric Cancer: A Systematic Review and MetaAnalysis. Clin Nutr (2019) 39(7):2045-54. doi: 10.1016/j.clnu.2019.10.021

4. Shachar SS, Williams GR, Muss HB, Nishijima TF. Prognostic Value of Sarcopenia in Adults With Solid Tumours: A Meta-Analysis and Systematic Review. Eur J Cancer (Oxf Engl 1990) (2016) 57:58-67. doi: 10.1016/ j.ejca.2015.12.030

5. Prado CM, Lieffers JR, McCargar LJ, Reiman T, Sawyer MB, Martin L, et al. Prevalence and Clinical Implications of Sarcopenic Obesity in Patients With Solid Tumours of the Respiratory and Gastrointestinal Tracts: A PopulationBased Study. Lancet Oncol (2008) 9(7):629-35. doi: 10.1016/S1470-2045(08) 70153-0

6. Cruz-Jentoft AJ, Bahat G, Bauer J, Boirie Y, Bruyère $\mathrm{O}$, Cederholm T, et al. Sarcopenia: Revised European Consensus on Definition and Diagnosis. Age Ageing (2019) 48(1):16-31. doi: 10.1093/ageing/afy169

7. Grossberg AJ, Chamchod S, Fuller CD, Mohamed AS, Heukelom J, Eichelberger $\mathrm{H}$, et al. Association of Body Composition With Survival and Locoregional Control of Radiotherapy-Treated Head and Neck Squamous Cell Carcinoma. JAMA Oncol (2016) 2(6):782-9. doi: 10.1001/ jamaoncol.2015.6339

8. Stone L, Olson B, Mowery A, Krasnow S, Jiang A, Li R, et al. Association Between Sarcopenia and Mortality in Patients Undergoing Surgical Excision of Head and Neck Cancer. JAMA Otolaryngol Head Neck Surg (2019) 145 (7):647-54. doi: 10.1001/jamaoto.2019.1185

9. Olson B, Edwards J, Stone L, Jiang A, Zhu X, Holland J, et al. Association of Sarcopenia With Oncologic Outcomes of Primary Surgery or Definitive Radiotherapy Among Patients With Localized Oropharyngeal Squamous Cell Carcinoma. JAMA Otolaryngol Head Neck Surg (2020) 146(8):714-22. doi: 10.1001/jamaoto.2020.1154

10. Achim V, Bash J, Mowery A, Guimaraes AR, Li R, Schindler J, et al. Prognostic Indication of Sarcopenia for Wound Complication After Total Laryngectomy. JAMA Otolaryngol Head Neck Surg (2017) 143(12):1159-65. doi: 10.1001/ jamaoto.2017.0547

11. Grote M, Maihöfer C, Weigl M, Davies-Knorr P, Belka C. Progressive Resistance Training in Cachectic Head and Neck Cancer Patients Undergoing Radiotherapy: A Randomized Controlled Pilot Feasibility Trial. Radiat Oncol (2018) 13(1):215. doi: 10.1186/s13014-018-1157-0

12. Sandmael JA, Bye A, Solheim TS, Stene GB, Thorsen L, Kaasa S, et al. Feasibility and Preliminary Effects of Resistance Training and Nutritional Supplements During Versus After Radiotherapy in Patients With Head and
Center under the The Image Guided Cancer Therapy (IGCT) Research Program, and grant and infrastructure support via the Charles and Daneen Stiefel Center for Head and Neck Cancer Oropharyngeal Cancer Research Program. CF has received direct industry grant/in-kind support, honoraria, and travel funding from Elekta $\mathrm{AB}$ unrelated to this project. Finally, we thank Haley Knapp-Berry for thoroughly proofreading this manuscript.

\section{SUPPLEMENTARY MATERIAL}

The Supplementary Material for this article can be found online at: https://www.frontiersin.org/articles/10.3389/fonc.2022.812159/ full\#supplementary-material

Neck Cancer: A Pilot Randomized Trial. Cancer (2017) 123(22):4440-8. doi 10.1002/cncr.30901

13. Lønbro S, Dalgas U, Primdahl H, Johansen J, Nielsen JL, Aagaard P, et al. Progressive Resistance Training Rebuilds Lean Body Mass in Head and Neck Cancer Patients After Radiotherapy-Results From the Randomized DAHANCA 25B Trial. Radiother Oncol (2013) 108(2):314-9. doi: 10.1016/ j.radonc.2013.07.002

14. Grossberg AJ, Rock CD, Edwards J, Mohamed ASR, Ruzensky D, Currie A, et al. Bioelectrical Impedance Analysis as a Quantitative Measure of Sarcopenia in Head and Neck Cancer Patients Treated With Radiotherapy. Radiother Oncol (2021) 159:21-7. doi: 10.1016/j.radonc.2021.03.005

15. Petak S, Barbu CG, Yu EW, Fielding R, Mulligan K, Sabowitz B, et al. The Official Positions of the International Society for Clinical Densitometry: Body Composition Analysis Reporting. J Clin Densitom (2013) 16(4):508-19. doi: 10.1016/j.jocd.2013.08.018

16. Leigheb M, de Sire A, Colangelo M, Zagaria D, Grassi FA, Rena O, et al. Sarcopenia Diagnosis: Reliability of the Ultrasound Assessment of the Tibialis Anterior Muscle as an Alternative Evaluation Tool. Diagn (Basel Switzerland) (2021) 11(11):2158. doi: 10.3390/diagnostics11112158

17. de Sire A, Baricich A, Renò F, Cisari C, Fusco N, Invernizzi M. Myostatin as a Potential Biomarker to Monitor Sarcopenia in Hip Fracture Patients Undergoing a Multidisciplinary Rehabilitation and Nutritional Treatment: A Preliminary Study. Aging Clin Exp Res (2020) 32(5):959-62. doi: 10.1007/ s40520-019-01436-8

18. Erlandson MC, Lorbergs AL, Mathur S, Cheung AM. Muscle Analysis Using pQCT, DXA and MRI. Eur J Radiol (2016) 85(8):1505-11. doi: 10.1016/ j.ejrad.2016.03.001

19. Amarasinghe KC, Lopes J, Beraldo J, Kiss N, Bucknell N, Everitt S, et al. A Deep Learning Model to Automate Skeletal Muscle Area Measurement on Computed Tomography Images. Front Oncol (2021) 11:580806. doi: 10.3389/ fonc.2021.580806

20. Paris MT, Tandon P, Heyland DK, Furberg H, Premji T, Low G, et al. Automated Body Composition Analysis of Clinically Acquired Computed Tomography Scans Using Neural Networks. Clin Nutr (2020) 39(10):3049-55. doi: 10.1016/j.clnu.2020.01.008

21. Kaplan SJ, Zhao KL, Koren M, Bentov I, Reed MJ, Pham TN. Thresholds and Mortality Associations of Paraspinous Muscle Sarcopenia in Older Trauma Patients. JAMA Surg (2020) 155(7):662-4. doi: 10.1001/jamasurg.2020.0435

22. Swartz JE, Pothen AJ, Wegner I, Smid EJ, Swart KM, de Bree R, et al. Feasibility of Using Head and Neck CT Imaging to Assess Skeletal Muscle Mass in Head and Neck Cancer Patients. Oral Oncol (2016) 62:28-33. doi: 10.1016/j.oraloncology.2016.09.006

23. Ufuk F, Herek D, Yüksel D. Diagnosis of Sarcopenia in Head and Neck Computed Tomography: Cervical Muscle Mass as a Strong Indicator of Sarcopenia. Clin Exp Otorhinolaryngol (2019) 12(3):317-24. doi: 10.21053/ceo.2018.01613

24. Bril SI, Wendrich AW, Swartz JE, Wegner I, Pameijer F, Smid EJ, et al. Interobserver Agreement of Skeletal Muscle Mass Measurement on Head and Neck CT Imaging at the Level of the Third Cervical Vertebra. Eur Arch Otorhinolaryngol (2019) 276(4):1175-82. doi: 10.1007/s00405-019-05307-w 
25. van Rijn-Dekker MI, van den Bosch L, van den Hoek JGM, Bijl HP, van Aken ESM, van der Hoorn A, et al. Impact of Sarcopenia on Survival and Late Toxicity in Head and Neck Cancer Patients Treated With Radiotherapy. Radiother Oncol (2020) 147:103-10. doi: 10.1016/j.radonc.2020.03.014

26. Yoshimura T, Suzuki H, Takayama H, Higashi S, Hirano Y, Tezuka M, et al. Prognostic Role of Preoperative Sarcopenia Evaluation of Cervical Muscles With Long-Term Outcomes of Patients With Oral Squamous Cell Carcinoma. Cancers (Basel) (2021) 13(18):4725. doi: 10.3390/cancers13184725

27. Charlson ME, Pompei P, Ales KL, MacKenzie CR. A New Method of Classifying Prognostic Comorbidity in Longitudinal Studies: Development and Validation. J Chronic Dis (1987) 40(5):373-83. doi: 10.1016/0021-9681 (87)90171-8

28. Mitsiopoulos N, Baumgartner RN, Heymsfield SB, Lyons W, Gallagher D, Ross R. Cadaver Validation of Skeletal Muscle Measurement by Magnetic Resonance Imaging and Computerized Tomography. J Appl Physiol (Bethesda Md 1985) (1998) 85(1):115-22. doi: 10.1152/jappl.1998.85.1.115

29. DeLong ER, DeLong DM, Clarke-Pearson DL. Comparing the Areas Under Two or More Correlated Receiver Operating Characteristic Curves: A Nonparametric Approach. Biometrics (1988) 44(3):837-45. doi: 10.2307/ 2531595

30. Tanabe C, Reed MJ, Pham TN, Penn K, Bentov I, Kaplan SJ. Association of Brain Atrophy and Masseter Sarcopenia With 1-Year Mortality in Older Trauma Patients. JAMA Surg (2019) 154(8):716-23. doi: 10.1001/jamasurg.2019.0988

31. Bozzetti F. Forcing the Vicious Circle: Sarcopenia Increases Toxicity, Decreases Response to Chemotherapy and Worsens With Chemotherapy. Ann Oncol (2017) 28(9):2107-18. doi: 10.1093/annonc/mdx271

32. Caan BJ, Cespedes Feliciano EM, Prado CM, Alexeeff S, Kroenke CH, Bradshaw P, et al. Association of Muscle and Adiposity Measured by Computed Tomography With Survival in Patients With Nonmetastatic Breast Cancer. JAMA Oncol (2018) 4(6):798-804. doi: 10.1001/ jamaoncol.2018.0137

33. Chamchod S, Fuller CD, Mohamed AS, Grossberg A, Messer JA, Heukelom J, et al. Quantitative Body Mass Characterization Before and After Head and Neck Cancer Radiotherapy: A Challenge of Height-Weight Formulae Using Computed Tomography Measurement. Oral Oncol (2016) 61:62-9. doi: 10.1016/j.oraloncology.2016.08.012

34. Jung AR, Roh JL, Kim JS, Choi SH, Nam SY, Kim SY. Efficacy of Head and Neck Computed Tomography for Skeletal Muscle Mass Estimation in Patients With Head and Neck Cancer. Oral Oncol (2019) 95:95-9. doi: 10.1016/ j.oraloncology.2019.06.009

35. Muresan BT, Sánchez Juan C, Artero A, Hernández Machancoses A, Almendros-Blanco P, Montoro A, et al. Diagnosis of Pre-Sarcopenia From a Single Selectional Crosscut at C3 Region, Using CT Scans Before Radiotherapy. Nutricion Hospitalaria (2019) 36(5):1101-8. doi: 10.20960/ nh. 02422

36. Bril SI, Chargi N, Wendrich AW, Wegner I, Bol GH, Smid EJ, et al. Validation of Skeletal Muscle Mass Assessment at the Level of the Third Cervical Vertebra in Patients With Head and Neck Cancer. Oral Oncol (2021) 123:105617. doi: 10.1016/j.oraloncology.2021.105617

37. Bril SI, van Beers MA, Chargi N, Carrillo Minulina N, Smid EJ, Dankbaar JW, et al. Skeletal Muscle Mass at C3 is a Strong Predictor for Skeletal Muscle Mass at L3 in Sarcopenic and Non-Sarcopenic Patients With Head and Neck Cancer. Oral Oncol (2021) 122:105558. doi: 10.1016/j.oraloncology.2021.105558

38. Yoon JK, Jang JY, An YS, Lee SJ. Skeletal Muscle Mass at C3 may Not be a Strong Predictor for Skeletal Muscle Mass at L3 in Sarcopenic Patients With
Head and Neck Cancer. PloS One (2021) 16(7):e0254844. doi: 10.1371/ journal.pone. 0254844

39. Bauer J, Morley JE, Schols A, Ferrucci L, Cruz-Jentoft AJ, Dent E, et al. Sarcopenia: A Time for Action. SCWD Position Paper J Cachexia Sarcopenia Muscle (2019) 10(5):956-61. doi: 10.1002/jcsm.12483

40. Montalvo RN, Counts BR, Carson JA. Understanding Sex Differences in the Regulation of Cancer-Induced Muscle Wasting. Curr Opin Support Palliat Care (2018) 12(4):394-403. doi: 10.1097/SPC.0000000000000380

41. Zhong X, Zimmers TA. Sex Differences in Cancer Cachexia. Curr Osteoporos Rep (2020) 18(6):646-54. doi: 10.1007/s11914-020-00628-w

42. Hetzler KL, Hardee JP, Puppa MJ, Narsale AA, Sato S, Davis JM, et al. Sex Differences in the Relationship of IL-6 Signaling to Cancer Cachexia Progression. Biochim Biophys Acta (2015) 1852(5):816-25. doi: 10.1016/ j.bbadis.2014.12.015

43. Olson B, Norgard MA, Levasseur PR, Zhu X, Marks DL. Physiologic and Molecular Characterization of a Novel Murine Model of Metastatic Head and Neck Cancer Cachexia. J Cachexia Sarcopenia Muscle (2021) 12(5):1312-32. doi: $10.1002 / \mathrm{jcsm} .12745$

44. Baracos VE, Reiman T, Mourtzakis M, Gioulbasanis I, Antoun S. Body Composition in Patients With Non-Small Cell Lung Cancer: A Contemporary View of Cancer Cachexia With the Use of Computed Tomography Image Analysis. Am J Clin Nutr (2010) 91(4):1133s-7s. doi: 10.3945/ajcn.2010.28608C

45. Anoveros-Barrera A, Bhullar AS, Stretch C, Esfandiari N, Dunichand-Hoedl AR, Martins KJB, et al. Clinical and Biological Characterization of Skeletal Muscle Tissue Biopsies of Surgical Cancer Patients. J Cachexia Sarcopenia Muscle (2019) 10(6):1356-77. doi: 10.1002/jcsm.12466

46. Kilgour RD, Vigano A, Trutschnigg B, Hornby L, Lucar E, Bacon SL, et al. Cancer-Related Fatigue: The Impact of Skeletal Muscle Mass and Strength in Patients With Advanced Cancer. J Cachexia Sarcopenia Muscle (2010) 1 (2):177-85. doi: 10.1007/s13539-010-0016-0

47. Burkart M, Schieber M, Basu S, Shah P, Venugopal P, Borgia JA, et al. Evaluation of the Impact of Cachexia on Clinical Outcomes in Aggressive Lymphoma. Br J Haematol (2019) 186(1):45-53. doi: 10.1111/bjh.15889

Conflict of Interest: Author YC is currently employed by Seagen Inc, although this employment resulted after data and analyses were completed.

The remaining authors declare that the research was conducted in the absence of any commercial or financial relationships that could be construed as a potential conflict of interest.

Publisher's Note: All claims expressed in this article are solely those of the authors and do not necessarily represent those of their affiliated organizations, or those of the publisher, the editors and the reviewers. Any product that may be evaluated in this article, or claim that may be made by its manufacturer, is not guaranteed or endorsed by the publisher.

Copyright ( 2022 Olson, Edwards, Degnin, Santucci, Buncke, Hu, Chen, Fuller, Geltzeiler, Grossberg and Clayburgh. This is an open-access article distributed under the terms of the Creative Commons Attribution License (CC BY). The use, distribution or reproduction in other forums is permitted, provided the original author $(s)$ and the copyright owner(s) are credited and that the original publication in this journal is cited, in accordance with accepted academic practice. No use, distribution or reproduction is permitted which does not comply with these terms. 